\section{Antídotos e medicamentos utilizados para tratar intoxicações no Brasil: necessidades, disponibilidade e oportunidades}

\author{
Antidotes and medicines used to treat poisoning \\ in Brazil: needs, availability and opportunities
}

\author{
Antídotos y medicamentos utilizados para \\ tratar intoxicaciones en Brasil: necesidades, \\ disponibilidad y oportunidades
}

Tais F. Galvão 1,2

Fabio Bucaretchi 3

Eduardo M. De Capitani 3

Maurício G. Pereira 1

Marcus T. Silva 4

\footnotetext{
${ }^{1}$ Faculdade de Medicina, Universidade de Brasília, Brasília, Brasil.

2 Hospital Universitário Getúlio Vargas, Universidade Federal do Amazonas, Manaus, Brasil.

3 Faculdade de Ciências Médicas, Universidade Estadual de Campinas, Campinas, Brasil.

${ }^{4}$ Faculdade de Medicina, Universidade Federal do Amazonas, Manaus, Brasil.

Correspondência T. F. Galvão

Faculdade de Medicina, Universidade de Brasília. Campus Universitário, conjunto 16, sala 77, Asa Norte. Brasília, DF 70910-900, Brasil. taisgalvao@gmail.com
}

\begin{abstract}
Antidotes and certain other drugs are essential for treating some types of poisoning. Failures in their supply can jeopardize the population's health and safety. The current study aimed to assess the availability of antidotes and other drugs used in the treatment of poisonings in Brazil. International guidelines were used as the basis for selecting 41 antidotes for analysis, none of which currently protected by patents. Of these, 27 are registered in Brazil, but 11 of these are available in inadequate forms for treating poisoning, leaving 16 commercially available antidotes. Only one-third of the drugs needed for treating poisoning are included in the country's list of essential drugs. The article also presents a proposal for supplying the demand for one of the antidotes, anti-digoxin antibody, considering Brazil's domestic capacity for manufacturing immunobiologicals. The study's results show the limitations to adequate treatment for poison victims in Brazil and reinforce the urgent need to strengthen public policies in this area.
\end{abstract}

Poisoning; Antidotes; Pharmaceutical Preparations; Pharmaceutical Services; Health Technology Assessment

\section{Resumo}

Antídotos e determinados medicamentos são essenciais ao tratamento de algumas intoxicações e não podem sofrer falhas no abastecimento, sob o risco de prejudicar a saúde e a segurança da população. O objetivo deste trabalho foi avaliar a disponibilidade de antídotos e medicamentos recomendados para o tratamento de intoxicações no Brasil. A partir de consensos internacionais, foram selecionados 41 antídotos para análise, todos sem patente em vigência. Desses, 27 são registrados, porém 11 estão disponíveis em formas inadequadas ao tratamento de intoxicações, restando 16 medicamentos comercialmente disponíveis. Somente um terço dos medicamentos necessários para o tratamento de intoxicações está incluído na relação de medicamentos essenciais do país. Em adição, é apresentada proposta de suprimento das demandas a um dos antídotos, anticorpo antidigoxina, considerando a capacidade de produção nacional de imunobiológicos. Os resultados demonstram limitação da assistência adequada aos pacientes intoxicados no país e reforçam a necessidade urgente de políticas públicas na área.

Envenenamento; Antídotos; Preparações Farmacêuticas; Assistência Farmacêutica; Avaliação de Tecnologias de Saúde 


\section{Introdução}

A maior parte das intoxicações pode ser clinicamente abordada apenas com tratamento de suporte. Algumas condições, entretanto, requerem a administração de antídotos e medicamentos específicos, e a ausência desses nas unidades de emergência e serviços de referência representa importante fator limitante ao restabelecimento dos pacientes 1,2 . A disponibilidade de tais tecnologias é estratégica do ponto de vista de saúde pública e de segurança nacional ${ }^{3}$.

A despeito da importância dos antídotos e medicamentos usados em intoxicações, diagnósticos anteriores apontam para falhas no abastecimento desses em diferentes contextos 4,5,6,7,8,9. Vários fatores estão associados a esse cenário, incluindo o custo e a necessidade de manutenção de estoques mínimos, que frequentemente vêm a perder validade e precisam ser repostos 8 .

Para orientar o abastecimento desses insumos, consensos internacionais baseados em evidência foram desenvolvidos 5,10 resultando em melhor assistência aos pacientes intoxicados 11,12. Os Centros de Informações Toxicológicas (CIT), unidades especializadas em prover informações sobre o tratamento de intoxicações, prestam papel essencial nessa tarefa, principalmente fornecendo dados sobre a magnitude do problema 13. No Brasil, o relatório mais atual mostra que 25 dos 35 CIT do país atenderam cerca de 100 mil intoxicações em 2010, sendo os medicamentos o grupo de agentes mais frequente. Pelos dados fornecidos, não é possível conhecer as substâncias mais envolvidas, informação que auxiliaria no planejamento da assistência farmacêutica desse agravo.

Os CIT brasileiros possuem diferentes inserções institucionais, sendo ligados principalmente a universidades e a secretarias estaduais e municipais de saúde (Associação Brasileira de Centros de Informação e Assistência Toxicológica. Informações sobre os Centros de Informação e Assistência Toxicológica do Brasil. http://www.abracit. org.br/abracit_site $/$ index.php? option $=$ com wrapper\&view=wrapper\&Itemid=4, acessado em 15/Mai/2013). Muitos deles enfrentam dificuldades em obter recursos para sua manutenção, apesar de existirem evidências nacionais e internacionais que comprovam sua efetividade e viabilidade econômica 14,15. Em que pesem a importância dos dados dos CIT para vigilância sanitária e epidemiológica, esses são dependentes da qualidade da principal atividade que os CIT desempenham: assistência presencial ou à distância em caso de intoxicação ${ }^{13}$. Atendendo demandas da sociedade civil organizada, foi iniciado um grupo de trabalho no âmbito do Siste- ma Único de Saúde (SUS) para elaboração das diretrizes em toxicologia no SUS, incluindo a questão dos CIT e antídotos 16. Até o momento, entretanto, não há definição de uma política nacional de saúde pública na área.

O presente trabalho teve como objetivo avaliar a disponibilidade de antídotos e outros medicamentos recomendados para o tratamento de intoxicações no Brasil. A partir do diagnóstico obtido, foi possível identificar os produtos com dificuldades de acesso e apresentar uma proposta de solução para um dos antídotos.

\section{Método}

\section{Identificação dos medicamentos}

Considerando as inúmeras possibilidades de intoxicações, foi realizada uma revisão da relação dos antídotos e medicamentos necessários ao tratamento de intoxicações, orientada por listas de antídotos adotadas em outros países 17,18,19,20,21, definidas por instituições e sociedades científicas da área. Não foram considerados soros heterólogos para o tratamento de acidentes com animais peçonhentos, por já estarem inseridos e atendidos integralmente pelo Programa Nacional de Controle de Acidentes por Animais Peçonhentos 22.

Foi elaborada uma lista simples contendo todos os medicamentos presentes nas listas internacionais. Essa relação foi avaliada por dois autores, experientes na área de toxicologia clínica (F.B., E.M.D.C.), para confirmação da relevância dos medicamentos no país.

\section{Informações clínicas}

Todos os medicamentos foram classificados de acordo com o Anatomical Therapeutic Chemical Classification System (Norwegian Institute of Public Health, Collaborating Centre for Drug Statistics Methodology, World Health Organization. ATC/DDD index 2013. http://www.whocc. no/atc_ddd_index/, acessado em 15/Jan/2013). As indicações do uso dos medicamentos seguiram as definições dos consensos internacionais $17,18,19,20,21$.

Por meio de buscas nas bases de dados MEDLINE, UpToDate e Micromedex, foi identificado o delineamento epidemiológico da melhor evidência disponível sobre a eficácia ou efetividade do medicamento.

O tempo de acesso que o insumo deveria estar disponível para o tratamento de intoxicações foi extraído das recomendações existentes nas listas internacionais de antídotos 17,18,19,20,21, 
sendo classificados como: (1) de disponibilidade imediata (medicamentos que devem estar disponíveis no primeiro atendimento, em ambulâncias e unidades de emergência); (2) disponível em até 1 hora (medicamentos que devem estar disponíveis em unidades de emergência); (3) disponibilidade em estoque (medicamentos armazenados em hospitais de referência).

\section{Informações de acesso}

De posse da lista de medicamentos, os autores consultaram o registro de cada item na base da Agência Nacional de Vigilância Sanitária (ANVISA. Consulta de produtos: medicamentos. http://www.anvisa.gov.br/datavisa/consulta_ produto/Medicamentos/frmConsultaMedica mentos.asp, acessado em 15/Jan/2013) e verificaram a compatibilidade da forma farmacêutica registrada com a formulação indicada para tratar intoxicações.

Foi conferida a inclusão dos medicamentos nas relações da Organização Mundial da Saúde (OMS) para uso adulto e infantil 23,24 e na Relação Nacional de Medicamentos Essenciais (RENAME) 25.

Por fim, o perfil de proteção patentária de cada medicamento foi consultado nas bases de registro Orange Book do U.S. Food and Drug Administration (http://www.accessdata.fda. gov/scripts/cder/ob/default.cfm, acessado em 15/Jan/2013) e European Patent Office (http:// www.epo.org/searching.html, acessado em 15/ Jan/2013) a fim de se verificar impedimentos legais para a produção nacional.

\section{Avaliação dos medicamentos no âmbito nacional e recomendações}

Os dados coletados foram sumarizados para análise do perfil de cada produto, sendo confrontadas as informações clínicas e de acesso para identificar os insumos cuja produção nacional é necessária e oportuna.

A partir desses resultados, elegeu-se um dos medicamentos para propor um cenário envolvendo incentivo à produção nacional, visando resolver a disponibilidade desse insumo no SUS. O cenário foi apresentado seguindo os itens: (1) informações epidemiológicas e de magnitude do agravo principal coberto pela tecnologia; (2) evidências sobre o uso da tecnologia; e (3) capacidade instalada no país para o desenvolvimento do produto.

\section{Resultados}

\section{Seleção dos medicamentos}

A partir das listas internacionais, foram identificados 43 medicamentos. Após avaliação das informações clínicas 26 , dois foram excluídos da análise, resultando em 41 medicamentos analisados quanto ao acesso no país (Figura 1).

Dos 41 medicamentos analisados, 27 possuem autorização para comercialização concedida pela ANVISA. Entretanto, 11 desses estão disponíveis em formas farmacêuticas ou em apresentações inadequadas ao tratamento de intoxicações. O que resultou em 16 medicamentos comercialmente disponíveis para o tratamento de intoxicações. Todos os 41 medicamentos analisados estão livres de patente.

A principal indicação no tratamento de intoxicações está descrita na Tabela 1. A maior parte dos medicamentos pertence ao grupo "V - vários" da ATC e deve estar disponível, de forma imediata, para uso e sua evidência primária é baseada em estudos observacionais (Tabela 2).

Cerca de um terço dos medicamentos necessários para o tratamento de intoxicações está incluído na RENAME, e menos da metade está prevista nas listas da OMS.

\section{Produtos com dificuldade de acesso}

A Tabela 3 apresenta os 25 medicamentos não disponíveis em apresentações comerciais no país. A maior parte deles está disponível somente por importação 27,28 ou é comercializada associada a outros produtos ou em apresentações não adequadas para o tratamento de intoxicações. Por exemplo, a acetilcisteína (solução injetável $3 \mathrm{mg} / \mathrm{mL}$ ) e a atropina (solução injetável 0,25mg/ $\mathrm{mL}$ ) são comercializadas em concentrações muito abaixo do recomendado para o tratamento de intoxicação (acetilcisteína: solução injetável de 200mg/mL em apresentações de 30mL; soluções para uso por via oral ou tubagem nasogástrica, em apresentações de $30 \mathrm{~mL}$ a $20 \%$; atropina: solução injetável 1mg/mL) 17,18,19,20,21. O carvão ativado, adsorvente utilizado em intoxicações por ingestão de algumas substâncias, é disponível em comprimidos de $250 \mathrm{mg}$, enquanto que, para o tratamento de intoxicações, deve ser usado na forma de pó, em doses de acordo com o peso do paciente, geralmente de $1 \mathrm{~g} / \mathrm{kg}$ 17,18,19,20,21.

Para contornar a falta de antídotos e de outros insumos necessários ao tratamento de intoxicações, hospitais e CIT mais estruturados adquirem os medicamentos por meio de manipulação por farmácias magistrais. É particularmente comum a manipulação dos seguintes 


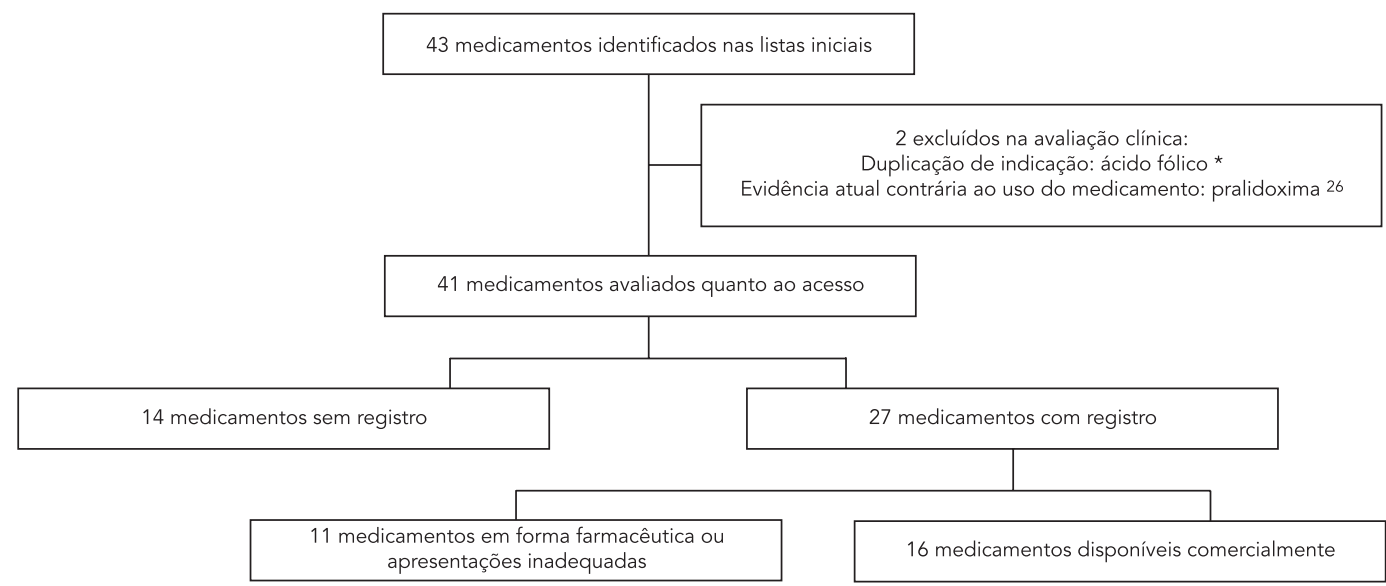

* O ácido fólico indicado pelos consensos é na forma injetável, não disponível no Brasil. Optou-se por incluir apenas o ácido folínico que já está disponível na forma recomendada.

itens: álcool absoluto, azul de metileno, carvão ativado, nitrito de sódio, solução de polietilenoglicol e tiossulfato de sódio. Outros insumos que requerem tecnologias específicas para produção, como o anticorpo antidigoxina e o nitrito de amila, podem ser obtidos por importação direta, seguindo processo de compra específico em cada instituição, em geral de difícil execução. Em alguns casos, há necessidade de importação de princípios ativos 27,28 por farmácias magistrais, como o dimercaprol, o ácido dimercaptosuccínico (succímer) e edetato cálcico dissódico, por exemplo, para então proceder à manipulação farmacêutica.

\section{Cenário selecionado: anticorpo antidigoxina}

- Informações epidemiológicas e de magnitude do agravo principal coberto pela tecnologia

Os glicosídeos cardiogênicos (digoxina, digitoxina, deslanósido) são indicados para o tratamento de insuficiência cardíaca e para o controle da taxa de resposta do ventrículo em pacientes com fibrilação atrial 29. Algumas plantas como a Digitalis lanata, Nerium oleander e Thevetia peruviana também possuem glicosídeos cardiogênicos 30 . É característica de tais substâncias ter índice terapêutico estreito, sendo comum a toxicidade em pequenas doses 31 .
O tratamento de primeira linha recomendado nas intoxicações graves é feito com fragmento de anticorpo (Fab) antidigoxina, objetivando a correção de hipocalemia, bradicardia, ectopia ventricular e disritmias 30,31. Na ausência desse medicamento, é indicado o uso de atropina e de marca-passo dentre outras terapias com efetividade muito menor 30 .

Não estão disponíveis dados epidemiológicos nacionais sobre as intoxicações por glicosídeos cardiogênicos. Os dados disponíveis apontam apenas que os medicamentos, como um todo, representam cerca de um terço do total das intoxicações em 2010 (Sistema Nacional de Informações Toxico Farmacológicas, Fundação Oswaldo Cruz. Casos registrados de intoxicação e/ou envenenamento. http://www.fio cruz.br/sinitox_novo/cgi/cgilua.exe/sys/start. htm?sid=379, acessado em 21/Jan/2013). Nesse mesmo ano, nos Estados Unidos, foram registrados 2.458 casos de intoxicações por medicamentos com glicosídeos cardiogênicos, sendo 166 casos graves e 32 óbitos 32 . É esperado incremento nos casos de exposição a compostos digitálicos no Brasil, em especial devido à transição epidemiológica e ao aumento nos casos de insuficiência cardíaca requerendo tratamento farmacológico 33,34. Em países desenvolvidos, a despeito da redução de consumo de medicamentos digitálicos, a frequência de intoxicação é crescente 35 . 
Medicamentos e principal indicação considerada na análise.

Medicamentos [forma farmacêutica]

Acetilcisteína (NAC) [injetável]

Anticorpo antidigoxina [injetável]

Atropina [injetável]

Bicarbonato de sódio [injetável]

Carvão vegetal ativado [pó]

Ciproeptadina [comprimido]

Cloreto de cálcio [injetável]

Cloreto de metiltionínio (azul de metileno) [injetável]

Dantroleno [injetável]

Desferroxamina [injetável]

Diazepam [injetável]

Difenidramina [injetável]

Dimercaprol (DMPS) [injetável]

Dinitrato de isossorbida [injetável]

Edetato dicobáltico (EDTA dicobáltico) [injetável]

Edetato cálcio dissódico [injetável]

Etanol (álcool absoluto) [injetável]

Fentolamina [injetável]

Folinato de cálcio (ácido folínico) [injetável]

Fisostigmina [injetável]

Flumazenil [injetável]

Fomepizol [injetável]

Glicose 50\% [injetável]

Glucagon [injetável]

Gluconato de cálcio [injetável, gel]

Hexacianoferrato férrico (azul da Prússia) [injetável]

Hidroxicobalamina [injetável]

lodeto de potássio [comprimido]

Isoproterenol [injetável]

Kit cianeto (nitrito de amila - inalatório; nitrito de sódio injetável; tiossulfato de sódio) [injetável]

Mesna [injetável]

Naloxona [injetável]

Octreotida [injetável]

Penicilamina [comprimido]

Pentetato cálcio trisódico (cálcio DTPA) [injetável]

Piridoxina [injetável]

Polietilenoglicol [enema (solução eletroliticamente balanceada)]

Prociclidina [injetável]

Protamina [injetável]

Succímer (DMSA) [comprimido]

Vitamina K1 (fitomenadiona) [injetável]
Principal indicação (nos casos de intoxicação por determinado agente, apenas o nome da substância é apresentado)

Paracetamol

Esteroides cardioativos (glicosídeos digitálicos)

Inibidores da acetilcolinesterase

Antidepressivos tricíclicos e outras drogas com efeito semelhante à quinidina

(alcalinização sérica); salicilatos e fenobarbital (alcalinização urinária)

Adsorvente para descontaminação gastrointestinal

Síndrome serotoninérgica

Betabloqueadores, bloqueadores de canal de cálcio

Agentes metemoglobinizantes

Síndrome neuroléptica maligna

Sais de ferro

Tratamento de convulsões, agitação e para precordialgia (p.ex.: cocaína e anfetaminas)

Distonia aguda desencadeada por bloqueadores dopaminérgicos

(p.ex.: metoclopramida, butirofenonas e fenotiazínicos)

Mercúrio, arsênio, ouro

Anfetaminas, fenilciclidina e cocaína (hipertensão)

Cianeto

Metais (principalmente chumbo)

Metanol; etilenoglicol

Medicamentos simpatomiméticos, inibidores da MAO e clonidina

(hipertensão resistente)

Metotrexato; metanol; ácido fórmico

Agentes anticolinérgicos

Benzodiazepínicos

Metanol; etilenoglicol

Hipoglicemiantes (ex: sulfonilureias)

Betabloqueadores; bloqueadores de canal de cálcio; antidepressivos tricíclicos

Bloqueadores de canal de cálcio; ácido fluorídrico (casos graves: injeção intra-arterial

próxima ao local do contato)

Tálio; césio

Cianeto

lodo radioativo

Verapamil; cloroquina; propranolol

Cianeto

Ciclofosfamida

Opioides

Hipoglicemiantes orais (p.ex.: sulfonilureias)

Mercúrio; níquel; chumbo; arsênio; cobre

Plutônio; amerício; cromo

Isoniazida; hidralazina e derivados

Irrigação intestinal nas intoxicações por comprimidos de sais de ferro, lítio e na ingestão de envelopes de cocaína ou heroína para tráfico de drogas ("mulas")

Síndrome de liberação extrapiramidal

Heparina

Chumbo; arsênio; mercúrio

Anticoagulantes cumarínicos 
Características dos medicamentos usados em intoxicações $(n=41)$.

\begin{tabular}{|c|c|c|}
\hline Características & $\mathrm{n}$ & $\%$ \\
\hline \multicolumn{3}{|l|}{ Classificação anatômico-terapêutica (código) 20} \\
\hline Vários (V) & 23 & 56 \\
\hline Aparelho digestivo e metabolismo (A) & 4 & 10 \\
\hline Sangue e órgãos hematopoiéticos (B) & 3 & 7 \\
\hline Aparelho cardiovascular (C) & 2 & 5 \\
\hline Preparações hormonais sistêmicas, excluindo hormônios sexuais e insulinas $(H)$ & 2 & 5 \\
\hline Sistema musculoesquelético (M) & 2 & 5 \\
\hline Sistema nervoso (N) & 2 & 5 \\
\hline Aparelho respiratório (R) & 2 & 5 \\
\hline Medicamentos dermatológicos (D) & 1 & 2 \\
\hline \multicolumn{3}{|l|}{ Recomendação de disponibilidade no serviço } \\
\hline Imediata (ambulâncias e unidades de emergência) & 19 & 46 \\
\hline Em 1 hora (unidades de emergência) & 15 & 37 \\
\hline Estoque (estoques estratégicos em hospital de referência) & 6 & 15 \\
\hline Não definida & 1 & 2 \\
\hline \multicolumn{3}{|l|}{ Delineamento da melhor evidência disponível } \\
\hline Ensaio clínico randomizado & 6 & 15 \\
\hline Estudo observacional controlado & 12 & 29 \\
\hline Estudo observacional não-controlado & 21 & 51 \\
\hline Modelos animais & 1 & 2 \\
\hline Opinião de especialistas & 1 & 2 \\
\hline Registrados na ANVISA & 27 & 66 \\
\hline Disponíveis no mercado brasileiro em formas farmacêuticas inadequadas & 11 & 27 \\
\hline Livres de proteção patentária & 41 & 100 \\
\hline Disponível na RENAME 2012 & 12 & 29 \\
\hline Disponível na lista de medicamentos essenciais da OMS & 17 & 41 \\
\hline
\end{tabular}

ANVISA: Agência Nacional de Vigilância Sanitária; OMS: Organização Mundial da Saúde; RENAME: Relação Nacional de Medicamentos Essenciais.

\section{- Evidências sobre o uso da tecnologia}

Uma revisão sistemática da Colaboração Cochrane aponta que o uso de anticorpo antidigoxina reduz significativamente a mortalidade nos casos de intoxicação por glicosídeos digitálicos (RR = 0,60; IC95\%: 0,44-0,81) 36 .

Quanto aos custos, estão disponíveis duas análises de custo-efetividade sobre o uso do anticorpo antidigoxina em intoxicações digitálicas, ambas realizadas nos Estados Unidos e mostrando a intervenção como custo-efetiva. A primeira limitou-se a casos graves em adultos e em crianças, e observou que o tratamento com anticorpo antidigoxina reduz a probabilidade de morrer nos pacientes mais graves, sendo estimado custo de 1.900 a 5.400 dólares por vida 37. Quando o anticorpo é usado nos pacientes menos graves, os gastos totais diminuem devido à redução no tempo de internação e menos uso de tratamentos agressivos como a implantação de marcapasso. O outro estudo disponível avaliou casos não graves por meio de um modelo de decisão, sendo observado gasto total adicional de 54 dólares com a tecnologia avaliada comparada à terapia padrão, e foi estimada redução do tempo de internação em $72 \%$ dos casos 38 . 
Antídotos e outros medicamentos usados no tratamento de intoxicações com dificuldade de acesso no Brasil $(n=25)$.

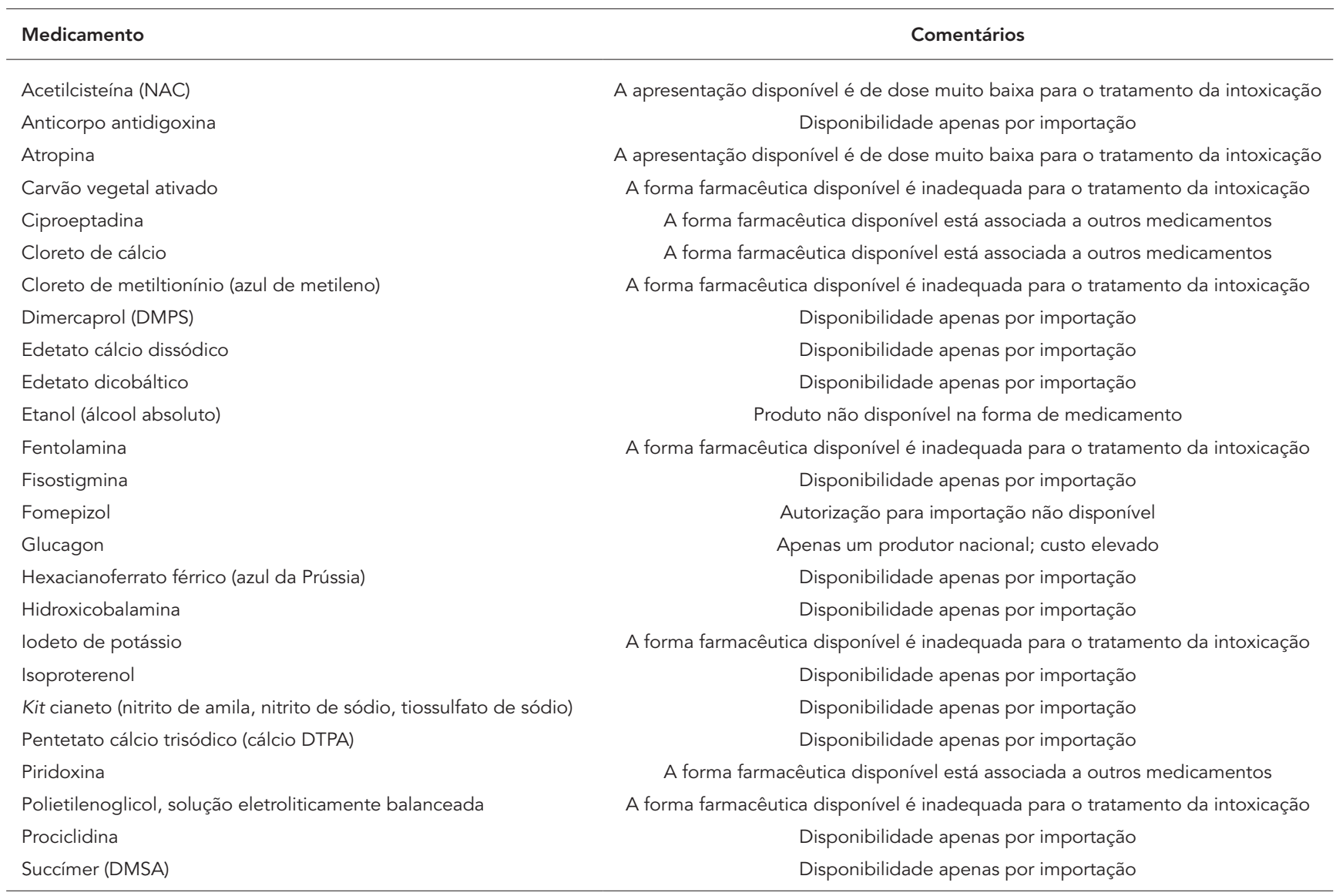

\section{- Capacidade instalada no país para o desenvolvimento do produto}

O Brasil domina a tecnologia de produção em escala de diversos imunobiológicos, incluindo os soros heterólogos antiveneno tipo $\mathrm{F}(\mathrm{ab})_{2}$ para tratamento de pacientes picados por animais peçonhentos, com padrão de qualidade reconhecido pela OMS, que serve outros países por meio de exportação 39,40 . Os principais institutos produtores no Brasil são o Instituto Butantan, em São Paulo; a Fundação Ezequiel Dias, em Minas Gerais; o Instituto Vital Brazil, no Rio de Janeiro; e o Centro de Produção e Pesquisa em Imunobiológicos, no Paraná.

Recente pesquisa sobre a produção e caracterização de anticorpo antidigoxina monoclonal (fragmento Fab) no país demonstram resultados promissores, apontando para a factibilidade da produção nacional do antídoto 41,42 .

\section{Discussão}

Nossa revisão apontou que pouco mais de um terço dos antídotos e outros medicamentos recomendados para o tratamento de intoxicações estão em formas farmacêuticas adequadas e comercialmente disponíveis no mercado brasileiro. A maior parte dos produtos requer importação ou é comercializada em apresentações impróprias à terapia. Esses achados indicam que a qualidade da assistência aos pacientes intoxicados no país está comprometida.

Foi proposta a resolução do acesso a um dos antídotos, o anticorpo antidigoxina. Além de suprir o mercado interno a um menor custo, o medicamento poderia ser exportado, atendendo demandas do mercado internacional, especialmente após a descontinuação da produção por um dos laboratórios 43. Atualmente, há somente um produtor mundial 44. Devido à ausência de concorrência, os preços praticados seguem a regra de monopólio. 
Se, por um lado, a situação de produção, disponibilidade e distribuição de antídotos no país é preocupante, por outro, a falta de dados epidemiológicos confiáveis pode passar a ideia de que intoxicação é um agravo de pouco impacto em saúde pública. Contribui para isso a ausência de dados padronizados sobre a incidência de intoxicações no Brasil 45 , o que pode ser interpretado como ausência do problema de saúde. Um estudo recente avaliou a tendência das mortes por intoxicações causadas por medicamentos e observou que, em uma década, houve aumento de $15 \%$ nos anos potenciais de vida perdidos, enquanto que, por todas as outras causas de óbito, ocorreu redução 46 . Vale ressaltar que tais resultados foram obtidos a partir de um desfecho raro em intoxicações, o óbito 47 .

O tratamento de intoxicações requer outros insumos farmacêuticos básicos para emergências, como, por exemplo, solução salina a $0,9 \%$ e oxigênio, que não compuseram o rol de itens analisados. Os medicamentos selecionados no presente estudo são considerados essenciais pelos consensos internacionais disponíveis e contemplam os itens necessários para prestar assistência farmacêutica em intoxicações 17,18,19,20,21. Não foi objeto do nosso estudo avaliar o custo das tecnologias em saúde, que é um fator que pode limitar fortemente o acesso. Futuros estudos devem incluir os custos e possivelmente apontarão mais pontos críticos na assistência farmacêutica. O glucagon, por exemplo, é um medicamento disponível no país, não requer importação, e o frasco de $1 \mathrm{mg}$ custa 45 dólares (ANVISA. Listas de preços de medicamentos. http://s.anvisa.gov. $\mathrm{br} / \mathrm{wps} / \mathrm{s} / \mathrm{r} / \mathrm{bs}$, acessado em 28/Jan/2013). Para tratar intoxicação grave por bloqueadores do canal de cálcio, são necessários de 5 a 15 frascos de $1 \mathrm{mg} 48$, resultando em quantidade de recursos considerável somente para fornecimento do insumo para o tratamento.

Outra limitação do presente estudo é a ausência de dados epidemiológicos nacionais que norteassem a inclusão de outros insumos importantes e que não fazem parte das listas internacionais. A disponibilização de informações sanitárias sobre legislação e registros de produtos no país é fragmentada e passível de estar desatualizada, o que pode ter distorcido parte dos nossos resultados. Diante dessa fragilidade, a definição dos potenciais medicamentos necessários no contexto brasileiro teve que ser feita por opinião de especialistas na área e não por evidência epidemiológica que desse robustez aos resultados.

O presente trabalho não avaliou a qualidade da evidência de cada tecnologia incluída, baseando-se somente nos resultados de consensos anteriores 17,18,19,20,21. Devido a limitações éticas e práticas de conduzir ensaios clínicos randomizados em situação de intoxicação aguda, a maior parte das informações científicas em toxicologia é proveniente de estudos não controlados, principalmente relatos e séries de casos ${ }^{49}$. Esse foi um confundimento que não pudemos controlar na análise.

As dificuldades em pesquisa na área podem explicar a escassez de medicamentos específicos para o tratamento de intoxicação. Soma-se a isso a falta de interesse econômico em investir em tecnologias para eventos raros, o que permite classificar os antídotos como produtos “órfãos" e usufruir de vantagens comerciais 50 . Cabe aos governos negociar e garantir o suprimento desses medicamentos essenciais. Nos Estados Unidos, por exemplo, é concedido registro prioritário a alguns antídotos, com exclusividade de mercado ao fabricante, como o caso do medicamento Cyanokit (hidroxicobalamina) (U.S. Food and Drug Administration. Orange Book: approved drug products with therapeutic equivalence evaluations. http://www.accessdata.fda.gov/ scripts/cder/ob/default.cfm, acessado em 15/ Jan/2013), tendo, como contrapartida, a garantia de abastecimento nacional pelo laboratório. Seguindo algumas estratégias, o efeito global sobre o orçamento de despesa das farmácias hospitalares pode ser minimizado. Antídotos de alto custo, que, em geral, são poucos (p.ex.: anticorpo antidigoxina, desferroxamina, fomepizol, hidroxicobalamina e glucagon), poderiam ser trocados junto aos produtores quando próximos do prazo de vencimento, seguindo a sistemática de compras consignadas, como já é realizado no Canadá, ou disponibilizados para outros serviços regionais de maior demanda 9,17 .

No Brasil, além de não haver garantia de acesso por parte do estado a esses insumos estratégicos, as instituições que fornecem assistência na área têm que desempenhar logística complexa e convencimento dos gestores da instituição - como a importação e manipulação de medicamentos - para viabilizar a assistência farmacêutica dos produtos.

Futuras pesquisas passam pelo investimento regular nos CIT e no registro padronizado, de preferência em tempo real, dos dados de atendimentos desses serviços, fornecendo informações epidemiológicas e sobre a efetividade dos medicamentos empregados nos tratamentos instituídos 13. É importante o desenvolvimento de novos antídotos, utilizando técnicas inovadoras 51 . No processo de desenvolvimento ou aprimoramento de tecnologias farmacêuticas estratégicas, as bases de dados de patentes podem ser fontes de informação úteis sobre produtos já existentes. 
Atualmente, por exemplo, há uma formulação de acetilcisteína que diminui os excipientes quelantes, induzindo melhor tolerância ao tratamento da intoxicação por paracetamol 52 .

\section{Conclusão}

O presente estudo aponta falhas de mercado e de políticas de saúde pública relacionadas à assistência farmacêutica de antídotos e medicamentos usados no tratamento de intoxicações no Brasil. Caso seja priorizada a resolução desse problema, o estudo indica caminhos que podem ser seguidos. Além da necessidade de se estruturar um sistema de informação de registros de casos de intoxicação, pesquisas na área de produção de medicamentos precisam ser incentivadas de modo a disponibilizar produtos úteis para o tratamento de intoxicações. A protelação, a fragmentação das responsabilidades e a improvisação nessa área precisam ser combatidas. Uma política que se antecipe a eventos de grande comoção ou calamidade em saúde pública se faz premente.

\section{Resumen}

Los antídotos y determinados medicamentos son esenciales para el tratamiento de algunas intoxicaciones, y su disponibilidad no puede fallar, o la salud y la seguridad de la población se ponen en peligro. Este estudio tuvo como objetivo evaluar la disponibilidad de antídotos y fármacos recomendados para el tratamiento por intoxicaciones en Brasil. Se seleccionaron para el análisis 41 antídotos de reconocido consenso internacional, todos ellos sin patentes en vigor. Veintisiete estaban registrados en Brasil, pero 11 se venden en preparados farmacéuticos, no apropiados para el tratamiento de intoxicaciones, lo que da como resultado 16 medicamentos disponibles en el mercado. Sólo se incluyen en la lista brasileña de medicamentos esenciales un tercio de los medicamentos necesarios para tratar intoxicaciones. Además, se presenta una propuesta para suplir uno de los antídotos, anticuerpo antidigoxina, considerando la capacidad de producción nacional. Los resultados muestran limitaciones en una atención adecuada a los pacientes intoxicados en Brasil y refuerzan la necesidad urgente de fortalecer las políticas públicas en este ámbito.

Envenenamiento; Antídotos; Preparaciones Farmacéuticas; Servicios Farmacéuticos; Evaluación de Tecnologías de Salud

\section{Colaboradores}

T. F. Galvão, M. G. Pereira e M. T. Silva participaram do planejamento do estudo, coleta, análise e interpretação dos dados, redação e revisão crítica do artigo e aprovação da versão final. F. Bucaretchi e E. M. De Capitani colaboraram na coleta, análise e interpretação dos dados, redação e revisão crítica do artigo e aprovação da versão final.

\section{Conflito de interesses}

Não declarado 


\section{Referências}

1. Smith SW. Drugs and pharmaceuticals: management of intoxication and antidotes. EXS 2010; 100:397-460.

2. Smollin CG. Toxicology: pearls and pitfalls in the use of antidotes. Emerg Med Clin North Am 2010; 28:149-61.

3. Rodgers GC, Condurache CT. Antidotes and treatments for chemical warfare/terrorism agents: an evidence-based review. Clin Pharmacol Ther 2010; 88:318-27.

4. Dart RC, Stark Y, Fulton B, Koziol-McLain J, Lowenstein SR. Insufficient stocking of poisoning antidotes in hospital pharmacies. JAMA 1996; 276:1508-10.

5. Bailey B, Bussières JF. Antidote availability in Quebec hospital pharmacies: impact of N-acetylcysteine and naloxone consumption. Can J Clin Pharmacol 2000; 7:198-204.

6. Nissen LM, Wong KH, Jones A, Roberts DM. Availability of antidotes for the treatment of acute poisoning in Queensland public hospitals. Aust J Rural Health 2010; 18:78-84.

7. Plataki M, Anatoliotakis N, Tzanakis N, Assithianakis P, Tsatsakis AM, Bouros D. Availability of antidotes in hospital pharmacies in Greece. Vet Hum Toxicol 2001; 43:103-5.

8. Marraffa JM, Cohen V, Howland MA. Antidotes for toxicological emergencies: a practical review. Am J Health Syst Pharm 2012; 69:199-212.

9. Gorman SK, Zed PJ, Purssell RA, Brubacher J, Willis GA. Antidote stocking in British Columbia hospitals. CJEM 2003; 5:12-7.

10. Dart RC, Goldfrank LR, Chyka PA, Lotzer D, Woolf AD, McNally J, et al. Combined evidence-based literature analysis and consensus guidelines for stocking of emergency antidotes in the United States. Ann Emerg Med 2000; 36:126-32.

11. Gair R. Antidote program in British Columbia. Am J Health Syst Pharm 2012; 69:1108.

12. Abbott V, Creighton M, Hannam J, Vincent T, Coulter C. Access in New Zealand to antidotes for accidental and intentional drug poisonings. J Prim Health Care 2012; 4:100-5.

13. Wolkin AF, Martin CA, Law RK, Schier JG, Bronstein AC. Using poison center data for national public health surveillance for chemical and poison exposure and associated illness. Ann Emerg Med 2012; 59:56-61.

14. Galvão TF, Silva MT, Silva CD, Barotto AM, Gavioli IL, Bucaretchi F, et al. Impact of a poison control center on the length of hospital stay of poisoned patients: retrospective cohort. São Paulo Med J 2011; 129:23-9.

15. Galvão TF, Silva EN, Silva MT, Bronstein AC, Pereira MG. Economic evaluation of poison centers: a systematic review. Int J Technol Assess Health Care 2012; 28:86-92.

16. Ministério da Saúde. Portaria no 298, de 9 de fevereiro de 2010. Institui Grupo de Trabalho para elaboração de diretrizes para as atividades das áreas da toxicologia no Sistema Único de Saúde (SUS). Diário Oficial da União 2010; 10 mar.
17. Bailey B, Bussières JF, Dumont M. Availability of antidotes in Quebec hospitals before and after dissemination of guidelines. Am J Health Syst Pharm 2003; 60:2345-9

18. Wiens MO, Zed PJ, Lepik KJ, Abu-Laban RB, Brubacher JR, Gorman SK, et al. Adequacy of antidote stocking in British Columbia hospitals: the 2005 Antidote Stocking Study. CJEM 2006; 8:409-16.

19. College of Emergency Medicine. Guideline on antidote availability for emergency departments. Appendix 1: stock levels \& storage recommendations. London: College of Emergency Medicine; 2011.

20. Dart RC, Borron SW, Caravati EM, Cobaugh DJ, Curry SC, Falk JL, et al. Expert consensus guidelines for stocking of antidotes in hospitals that provide emergency care. Ann Emerg Med 2009; 54:386-94.

21. British Columbia Drug and Poison Information Centre. Antidote stocking guidelines for British Columbia Hospitals British Columbia Drug \& Poison Information Centre. http://dpic.org/sites/de fault/files/Antidoteguide_Jun2012.pdf (acessado em 18/Jan/2013).

22. Fundação Nacional de Saúde. Manual de diagnóstico e tratamento de acidentes por animais peçonhentos. Brasília: Fundação Nacional de Saúde; 2001.

23. World Health Organization. WHO model list of essential medicines: 17th list. Geneva: World Health Organization; 2011.

24. World Health Organization. WHO model list of essential medicines for children: 3rd list. Geneva: World Health Organization; 2011.

25. Departamento de Assistência Farmacêutica e Insumos Estratégicos, Secretaria de Ciência, Tecnologia e Insumos Estratégicos, Ministério da Saúde. Relação Nacional de Medicamentos Essenciais: RENAME. Brasília: Ministério da Saúde; 2012.

26. Buckley NA, Eddleston M, Li Y, Bevan M, Robertson J. Oximes for acute organophosphate pesticide poisoning. Cochrane Database Syst Rev 2011; (2):CD005085.

27. Agência Nacional de Vigilância Sanitária. Resolução no 28, de 9 de maio de 2008. Autorizar a importação dos medicamentos constantes na lista de medicamentos liberados em caráter excepcional destinados unicamente a uso hospitalar ou sob prescrição médica, cuja importação esteja vinculada a uma determinada entidade hospitalar e/ou entidade civil representativa, para seu uso exclusivo, não se destinando à revenda ou ao comércio. Diário Oficial da União 2008; 12 mai.

28. Agência Nacional de Vigilância Sanitária. Resolução da Diretoria Colegiada - RDC no 81, de 5 de novembro de 2008. Dispõe sobre o regulamento técnico de bens e produtos importados para fins de vigilância sanitária. Diário Oficial da União 2008; 6 nov.

29. Feussner JR, Feussner DJ. Reassessing the efficacy of digitalis: from routine treatment to evidencebased medicine. Am J Med Sci 2010; 339:482-4. 
30. POISINDEX Managements. Cardiac glycosides. Greenwood Village: Truven Health Analytics; 2013.

31. Levine M, O'Connor A. Digitalis (cardiac glycoside) poisoning. In: Bason DS, editor. UpToDate. Waltham: UpToDate; 2012, p. 1-11.

32. Bronstein AC, Spyker DA, Cantilena LR, Green JL, Rumack BH, Dart RC. 2010 Annual Report of the American Association of Poison Control Centers' National Poison Data System (NPDS): 28th Annual Report. Clin Toxicol (Phila) 2011; 49:910-41.

33. Cubillos-Garzón LA, Casas JP, Morillo CA, Bautista LE. Congestive heart failure in Latin America: the next epidemic. Am Heart J 2004; 147:412-7.

34. Hernández-Leiva E. Epidemiología del síndrome coronario agudo y la insuficiencia cardiaca en Latinoamérica. Rev Esp Cardiol 2011; 64 Suppl 2:34-43.

35. Hussain Z, Swindle J, Hauptman PJ. Digoxin use and digoxin toxicity in the post-DIG trial era. J Card Fail 2006; 12:343-6.

36. Roberts DM, Buckley NA. Antidotes for acute cardenolide (cardiac glycoside) poisoning. Cochrane Database Syst Rev 2006; (4):CD005490.

37. Mauskopf JA, Wenger TL. Cost-effectiveness analysis of the use of digoxin immune Fab (ovine) for treatment of digoxin toxicity. Am J Cardiol 1991; 68:1709-14.

38. Di Domenico RJ, Walton SM, Sanoski CA, Bauman JL. Analysis of the use of digoxin immune fab for the treatment of non-life-threatening digoxin toxicity. J Cardiovasc Pharmacol Ther 2000; 577-85.

39. Gadelha CAG. A produção e o desenvolvimento de vacinas no Brasil. Hist Ciênc Saúde-Manguinhos 1996; 3:111-32.

40. Gadelha C, Azevedo N. Inovação em vacinas no Brasil: experiência recente e constrangimentos estruturais. Hist Ciênc Saúde-Manguinhos 2003; 10:697-724.

41. Murata VM. Produção e caracterização da porção Fab do anticorpo antidigoxina utilizando a tecnologia de phage display [Dissertação de Mestrado]. São Paulo: Universidade de São Paulo; 2012.

42. Murata VM, Schmidt MC, Kalil J, Tsuruta LR, Moro AM. Anti-digoxin Fab variants generated by phage display. Mol Biotechnol 2013; 54:269-77.

43. GlaxoSmithKline. Discontinuation of digibind. http://hcp.gsk.co.uk/news/discontinuation-ofdigibind.html (acessado em 28/Jan/2013).
44. BTG International. Specialty pharmaceuticals. http://www.btgplc.com/products/specialty-phar maceuticals (acessado em 28/Jan/2013).

45. Galvão TF, Pereira MG. Padronização das informações toxicológicas no Brasil. Ciênc Saúde Coletiva 2011; 16:3633-4.

46. Mota DM, Melo JR, Freitas DR, Machado M. Perfil da mortalidade por intoxicação com medicamentos no Brasil, 1996-2005: retrato de uma década. Ciênc Saúde Coletiva 2012; 17:61-70.

47. Lund C, Drottning P, Stiksrud B, Vahabi J, Lyngra M, Ekeberg I, et al. A one-year observational study of all hospitalized acute poisonings in Oslo: complications, treatment and sequelae. Scand J Trauma Resusc Emerg Med 2012; 20:49.

48. Barrueto F. Calcium channel blocker poisoning. In: Basow DS, editor. UpToDate. Waltham: UpToDate; 2012. p. 1-9.

49. Adami HO, Berry SC, Breckenridge CB, Smith LL, Swenberg JA, Trichopoulos D, et al. Toxicology and epidemiology: improving the science with a framework for combining toxicological and epidemiological evidence to establish causal inference. Toxicol Sci 2011; 122:223-34.

50. Steffen C. The dilemma of approving antidotes. Toxicology 2007; 233:13-9.

51. Szinicz L, Worek F, Thiermann H, Kehe K, Eckert S, Eyer P. Development of antidotes: problems and strategies. Toxicology 2007; 233:23-30.

52. Pavliv L, inventor; Cumberland Pharm Inc. (CUMB-Non-standard), assignee. Treating a patient having e.g. acetaminophen overdose, liver failure, cancers, methacrylonitrile poisoning and radio contrast-induced nephropathy, comprises administering a composition comprising acetylcysteine and a $\mathrm{pH}$ adjusting agent patent. United States patent US2,012,022,161-A1; WO2,012,012,640-A1; US2,012,022,161-A1. 2012 Jan 26.

Recebido em 14/Fev/2013

Versão final reapresentada em 26/Jun/2013

Aprovado em 30/Jul/2013 